\section{Therapie des malignen Melanoms}

\section{Überlebensvorteile durch zielgerichteten dualen Wirkansatz}

Der Kombinationstherapie gehört die Zukunft bei der Behandlung des fortgeschrittenen malignen Melanoms: Aktuelle Daten der coBRIM-Studie zeigen, dass sich das progressionsfreie Überleben (PFS) von Patienten mit BRAF $F^{V 600}$-Mutation-positivem malignem Melanom durch die zusätzliche Gabe von Cobimetinib zur Standardtherapie mit Zelboraf ${ }^{\circledR}$ (Vemurafenib) signifikant - im Median sogar um mehr als 5 Monate - verlängert [1]. Hintergrund der verbesserten Wirksamkeit sind synergistische Wirkeffekte der beiden Substanzen durch die gleichzeitige Blockade der BRAF- und MEK-Signalgebung in den Tumorzellen.

Mit Vemurafenib ist bereits seit über 3 Jahren ein Wirkstoff verfügbar, für den bei Patienten mit $B R A F^{V 600}$-Mutation-positivem malignem Melanom in Studien eine gute Antitumorwirkung mit verlängertem PFS und auch signifikanter Verlängerung des Gesamtüberlebens (OS) dokumentiert wird [2]. Der BRAF-Inhibitor stellte nach rund 30 Jahren ohne relevante therapeutische Neuerungen einen Durchbruch in der Melanomtherapie dar: Er verlängerte das OS der Patienten von 9,7 Monaten unter Dacarbazin auf median 13,6 Monate [2]. Mittlerweile wurden weltweit über 28000 Patienten mit Vemurafenib behandelt [3].

Cobimetinib - zusätzlicher Hebel zur Hemmung der Signalkaskade

Dennoch bleibt das fortgeschrittene maligne Melanom eine in der Regel schwer zu therapierende Erkrankung, da es im Verlauf der Behandlung zur Reaktivierung des für das Tumorwachstum entscheidenden MAPK-Signalwegs kommen kann. Die Kombinationstherapie Codes Tumorwachstums.

\section{Längstes bisher erreichtes PFS} tiert wurde. bimetinib plus Vemurafenib bietet mit Cobimetinib einen zusätzlichen Hebel, um die Signalkaskade zu unterbrechen und eine umfassende Blockade des MAPK-Signalwegs zu erreichen. Cobimetinib hemmt selektiv das Zellprotein MEK, das dem BRAF-Protein im MAPK-Signalweg nachgeordnet ist und ebenfalls die MAPKinasen aktiviert. Der duale Wirkansatz verstärkt so bei Patienten mit $B R A F^{V 600}$-Mutationpositivem malignem Melanom die Hemmung

Dass sich die kombinierte Therapie klinisch günstig auswirkt und die Prognose der Patienten verbessert, belegt eine aktuelle Auswertung der coBRIM-Studie, die erstmals auf der diesjährigen Jahrestagung der American Society of Clinical Oncology (ASCO) in Chicago präsen-

In der internationalen, randomisierten, doppelblinden und placebokontrollierten PhaseIII-Studie wurden 495 therapienaive Patienten mit $B R A F^{V 600}$-Mutation-positivem fortgeschrittenem Melanom mit Cobimetinib plus Vemurafenib oder mit Vemurafenib alleine behandelt. Dabei zeigte sich eine deutliche Überlegenheit des Kombinationsregimes: Das PFS lag unter alleiniger Behandlung mit Vemurafenib bei median 7,2 Monaten und wurde durch die zusätzliche Verabreichung von Cobimetinib auf median 12,3 Monate verlängert (Abb. 1). Die Kombinationsbehandlung mit dem MEK-Inhibitor steigert das PFS damit im Mittel um mehr als 5 Monate im Vergleich zur alleinigen Vemurafenib-Gabe, wodurch erstmals ein PFS von mehr als einem Jahr und insgesamt das

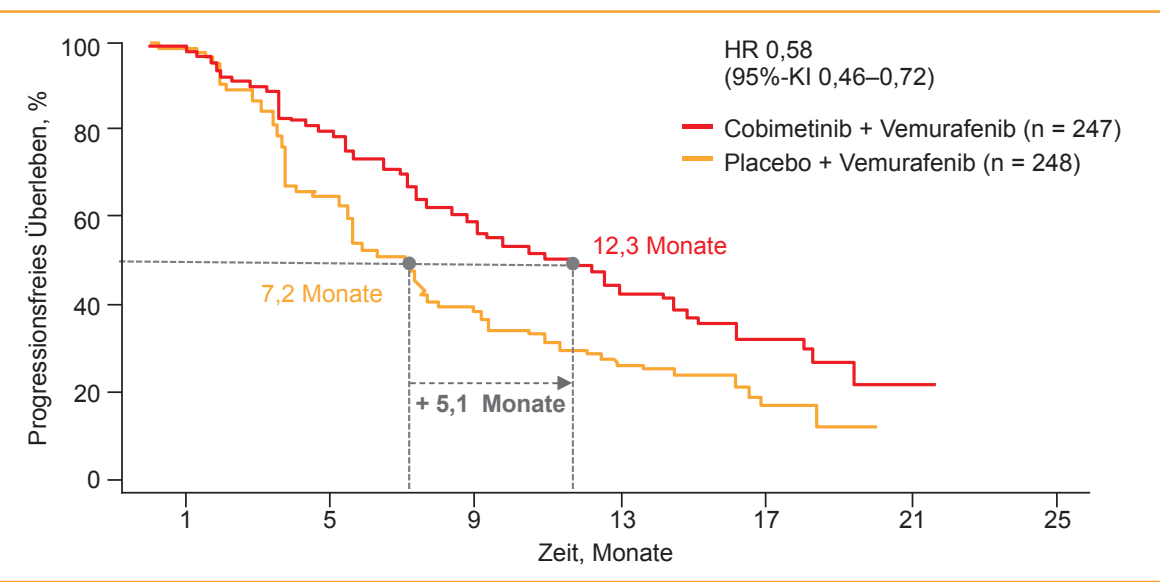

Abb. 1. coBRIM-Studie: PFS-Überlegenheit des Kombinationsregimes Cobimetinib plus Vemurafenib versus Vemurafenib alleine; modifiziert nach [1]. längste progressionsfreie Intervall bei diesem Patientenkollektiv erwirkt wurden.

Signifikante Überlebensvorteile und günstiges Sicherheitsprofil

Die auf dem diesjährigen ASCO präsentierten Daten der BRIM-7-Studie geben ebenfalls erste Anhaltspunkte für die klinische Wirksamkeit und das günstige Sicherheitsprofil des MEK-Inhibitors Cobimetinib [4]. In der Phase-1b-Studie wurden 129 Patienten mit einem $B R A F^{V 600}$-Mutation-positiven inoperablen, lokal fortgeschrittenen oder metastasierten Melanom mit Cobimetinib behandelt, wobei nach BRAF-Inhibitor-naiven und -vorbehandelten Patienten differenziert wurde. 61\% der Patienten, die zuvor keinen BRAF-Inhibitor erhalten hatten und mit der Kombination Cobimetinib plus Vemurafenib therapiert wurden, waren nach 2 Jahren noch am Leben. Die Überlebenszeit betrug in diesem Patientenkollektiv median 28,5 Monate. Im Hinblick auf das Sicherheitsprofil traten im Vergleich zwischen Kombinations- und Monotherapie keine wesentlichen Abweichungen auf. Die häufigsten Begleitreaktionen waren Durchfall, Übelkeit und Hautausschlag. Die Nebenwirkungen waren jedoch in aller Regel leicht bis moderat und vorübergehend.

\section{Fazit}

Die vorliegenden Studiendaten unterstreichen die Bedeutung der Kombinationstherapie bei fortgeschrittener Tumorerkrankung und zeigen, dass die duale Hemmung von BRAF und MEK bei Patienten mit fortgeschrittenem Melanom, bei denen eine $B R A F^{V 600}$-Mutation vorliegt, der Monotherapie in Hinblick auf das Ansprechen, das PFS und das OS überlegen ist. Die in der BRIM-7- und insbesondere in der coBRIM-Studie erzielten Therapieerfolge setzen somit die bereits mit Vemurafenib erwirkten Behandlungsfortschritte fort und berechtigen zu der Hoffnung, die Prognose von Patienten mit fortgeschrittenem Melanom künftig weiter verbessern zu können.

\section{Literatur}

1 Larkin J et al.: J Clin Oncol 2015;33(suppl): abstr 9006. 2 Chapman PB et al.: J Clin Oncol 2012;30(suppl): abstr 8502 .

3 PSUR (PBRER) vemurafenib. F. Hoffmann-La Roche Ltd; 13.10 .2014

4 Pavlick AC et al.: J Clin Oncol 2015;33(suppl): abstr 9020.

Weitere Informationen bei

Roche Pharma AG

Frau Ulla Satzger

ulla.satzger@roche.com 


\section{PharmaNews}

Karger Kompass Dermatologie 3 | 2 | 15

\section{Epiduo ${ }^{\circledR}$ - hochwirksam gegen Akne in der Langzeittherapie}

Die Ergebnisse der ELANG (Epiduo ${ }^{\circledR}$ in der Langzeitbehandlung mittelschwerer bis schwerer Akne mit und ohne Begleitmedikation)-Studie mit mehr als 5000 ausgewerteten Patienten bestätigen die hohe Wirksamkeit von Epiduo ${ }^{\circledR}$ (Adapalen 0,1\%/BPO 2,5\%; ADA-BPO) in der Behandlung der mittelschweren und schweren Akne. Über den 9-monatigen Studienverlauf verbesserte sich die Akne von einem mittleren $\mathrm{zu}$ einem leichten Akne-Schweregrad, wobei 25,8\% der Patienten am Studienende frei von Akne-Läsionen waren [1]. Die mittlere Lebensqualität verbesserte sich in Abhängigkeit vom initialen Schweregrad über die gesamte Therapiedauer [2]. Darüber hinaus war bei $84 \%$ der Patienten die Adhärenz auch unter Langzeittherapie gut [2]. Die Daten bestätigen die gute Verträglichkeit einer Langzeitbehandlung mit
Epiduo ${ }^{\circledR}$ : Primär milde Hautirritationen wurden bei $49,4 \%$ der Patienten dokumentiert, jedoch brachen lediglich 1,7\% der Patienten die Behandlung mit ADA-BPO aufgrund lokaler Hautirritationen ab [1]

\section{Epiduo ${ }^{\circledR}$ : Wirkstoffkombination ohne Antibiotika}

Die aktuellen Leitlinien der Deutschen Dermatologischen Gesellschaft zur Therapie der Akne empfehlen unter anderem die Kombination aus einem topischen Retinoid und BPO (wie in Epiduo ${ }^{\circledR}$ enthalten) bei mittelschwerer, entzündlicher Akne (Acne papulopustulosa) [3]. Das Retinoid Adapalen wirkt antientzündlich, unter anderem durch die Blockade von proinflammatorischen Toll-like Rezeptoren, und vermindert Mikrokomedonen - die noch nicht sichtbaren Vorläuferläsionen der klinisch sichtbaren Akne. Epiduo ${ }^{\circledR}$ ist frei von Antibiotika. Die Anwendungsdauer ist gemäß der Fachinformation unbegrenzt [4].

\section{Literatur}

1 Gollnick HPM et al.: J Eur Acad Dermatol Venereol 2015; 29 (Suppl 4):15-22.

2 Gollnick HPM et al.: J Eur Acad Dermatol Venereol 2015; 29 (Suppl 4):23-29.

3 Nast A et al.: J Dtsch Dermatol Ges 2010;8 (Suppl 2): 1-59; S2k-Leitlinie zur Behandlung der Akne; AWMFRegister Nr. 013/017.

4 Fachinformation Epiduo ${ }^{\circledR} 0,1 \% / 2,5 \%$ Gel; Stand: September 2014.

Weitere Informationen bei Galderma Laboratorium GmbH

Dr. Doris Porombka

Georg-Glock-Straße 8, 40474 Düsseldorf

doris.porombka@galderma.com

\section{Neue Phase-III-Daten zu Adalimumab bei Kindern und Jugendlichen} mit schwerer chronischer Plaque-Psoriasis

Im Rahmen des 23. World Congress of Dermatology (WCD) in Vancouver, Kanada, hat AbbVie die Ergebnisse einer neuen multizentrischen internationalen Phase-III-Studie vorgestellt. Die Daten bestätigen die im April durch die Europäische Kommission erteilte Zulassung von Adalimumab (Humira ${ }^{\circledR}$ ) zur Behandlung von Kindern und Jugendlichen ab 4 Jahren mit schwerer chronischer Plaque-Psoriasis, die nur unzureichend auf topische Therapien und Fototherapien angesprochen haben oder für die diese Therapien nicht geeignet sind.

Unter Adalimumab $(0,8 \mathrm{mg} / \mathrm{kg}$ alle 2 Wochen $)$ erreichten nach 16 Wochen signifikant mehr pädiatrische Patienten mit schwerer chronischer Plaque-Psoriasis einen Psoriasis Area Severity Index von 75 als unter Methotrexat (57,9 vs.
$32,4 \% ; \mathrm{p}=0,027)$. Deutlich mehr AdalimumabPatienten wiesen nach 16 Wochen einen PGAScore (PGA = Physicians Global Assessment) von $0 / 1$ (ein klares oder nahezu klares Hautbild) auf ( 60,5 vs. $40,5 \% ; \mathrm{p}=0,083)$ [1]. Zusätzlich erlangte ein hoher Prozentsatz an AdalimumabPatienten, bei denen nach Aussetzen der Therapie ein Verlust der Krankheitskontrolle beobachtet wurde, bei der 16-wöchigen Wiederholungsbehandlung mit Adalimumab einen PGAScore von 0/1 (55,6\%) [2]. Das Sicherheitsprofil unter Adalimumab war ähnlich wie unter Methotrexat.

"Psoriasis kann erhebliche physische und psychische Auswirkungen auf die betroffenen jungen Patienten haben", sagte Dr. Uwe Schwichtenberg, niedergelassener Dermatolo- ge in Bremen. "Diese Ergebnisse bestätigen, dass Adalimumab für Patienten mit schwerer pädiatrischer Plaque-Psoriasis, bei denen topische Therapien und Phototherapien nicht anschlagen, eine sichere und effektive Erstlinientherapie ist.»

\section{Literatur}

1 Papp K et al.: 23rd World Congress of Dermatology (WCD 2015), Vancouver, Canada, 2015; abstr 2970552.

2 Philipp S et al.: 23rd World Congress of Dermatology (WCD 2015), Vancouver, Canada, 2015; abstr 2970612.

Weitere Informationen bei

AbbVie Deutschland GmbH \& Co. KG

Jana Umbreit

Communication Manager Dermatologie

Mainzer Straße 8, 65189 Wiesbaden

Tel. +49 611 1720-4121

jana.umbreit@abbvie.com

Hyaluronidase in der ästhetischen Dermatologie: Indikationsspektrum hautnah präsentiert

Mit dem Trend zu ambulanten Operationen erweitert sich auch das Spektrum der Anwendungsgebiete von Hyaluronidase (Hylase ${ }^{\circledR}$ "Dessau») zur Unterstützung der Lokalanästhesie. Die Koapplikation des Enzyms zu einem Lokalanästhetikum (LA) sorgt für einen schnelleren Wirkeintritt des LA, eine VergröBerung des schmerzunempfindlichen Bereichs, weniger intra- und postoperative Schmerzen sowie eine höhere Patientenzufriedenheit [1, 2]. Typische Indikationen sind im Bereich der Dermatologie etwa die Entfernung von Lipomen, Atheromen, Warzen, Hämangiomen, Alterskeratosen und Fibromen sowie kosmetische Eingriffe wie Blepharoplastiken, die Entfernung von Abszessen oder auch die Cellulite-Behandlung [3-5]. «Bei kleineren chirurgischen Eingriffen wie Abszessen oder Keratosen ist der Zusatz des gewebeauflockernden Enzyms zu einem LA wie Lidocain aus unserer Praxis nich mehr wegzudenken. Von den Vorteilen profitieren sowohl unsere Patienten als auch wir als Operateure», fasste Dr. Tanja Fischer, Potsdam, zusammen.

\section{Quelle}

Praxistalk «Hylase ${ }^{\circledR}<$ Dessau $>$ hautnah - Indikationsspektrum in der ästhetischen Dermatologie», Veranstalter: RIEMSER Pharma GmbH, Potsdam, 30. April 2015

\section{Literatur \\ 1 Wohlrab J et al.: Dermatol Surg 2012;38:91-96.}

2 Wohlrab J et al.: Plast Reconstr Surg 2012;129:771e-772e

3 RIEMSER Arzneimittel AG, Potenzialstudie Hylase ${ }^{\circledR}$ "Dessau», Telefonumfrage bei 80 niedergelassenen Ärzten (40 Chirurgen und 40 Dermatologen), durchgeführt von emphasis Institut für Marktforschung im Gesundheitswesen $\mathrm{GmbH}$ im Auftrag de RIEMSER Arzneimittel AG, 2010.

4 Fratila A: Aesthet Dermatol 2013;2:2-6.

5 Fratila A: KosMed 2014;3:26-29.

\section{RIEMSER Pharma GmbH}

An der Wiek 7, 17493 Greifswald - Insel Riems

www.hylase.de 
Die Europäische Kommission hat den Anti-PD1Antikörper Pembrolizumab im Juli 2015 in der EU zur Behandlung des fortgeschrittenen metastasierten oder nichtresezierbaren malignen Melanoms zugelassen, sowohl im Rahmen einer FirstLine-Therapie als auch bei vorbehandelten Patienten. Die Zulassung basiert unter anderem auf den Daten der Phase-III-Studie KEYNOTE-006, in der Pembrolizumab gegenüber Ipilimumab einen Vorteil hinsichtlich des Gesamtüberlebens (OS), progressionsfreien Überlebens (PFS) und der Gesamtansprechrate gezeigt hatte. Die Studie wurde aufgrund der positiven Ergebnisse vorzeitig abgebrochen.

Wie Prof. Axel Hauschild, Kiel, bei der Launchpressekonferenz in München berichtete, hat die randomisierte, unverblindete, pivotale PhaseIII-Studie KEYNOTE-006 ihre 2 primären Endpunkte, das PFS und das OS, erreicht. In der Studie hatten 834 Patienten mit nicht resezierbarem fortgeschrittenem Melanom im Stadium III oder IV und maximal einer vorangegangenen Therapie entweder Pembrolizumab (KEYTRUDA ${ }^{\circledR}$ ) in einer Dosierung von $10 \mathrm{mg} / \mathrm{kg} \mathrm{KG}$ alle $2(\mathrm{n}=279)$ oder 3 Wochen $(\mathrm{n}=277)$ oder 4 Zyklen Ipilimumab $(3 \mathrm{mg} / \mathrm{kg}$ KG alle 3 Wochen; $\mathrm{n}=278$ ) erhalten.

In der ersten Interimsanalyse hatte sich gezeigt, dass die Gesamtansprechrate (nach RECISTKriterien) unter Pembrolizumab (alle 2 oder 3 Wochen verabreicht) mit 33,7 bzw. 32,9\% etwa dreimal so hoch war wie unter Ipilimumab $(11,9 \%)$. In der zweiten Interimsanalyse der Studie zeigte sich ein Überlebensvorteil für die Patienten, die mit Pembrolizumab behandelt worden waren. Das 1-Jahres-OS für die Patienten unter Pembrolizumab alle 2 Wochen lag bei $74,1 \%$ und alle 3 Wochen bei $68,4 \%$, während es für Patienten unter Ipilimumab bei $58,2 \%$ lag [1]. Die Hazard Ratio (HR) der bei- den Pembrolizumab-Gruppen im Vergleich zu Ipilimumab betrug 0,63 (2 Wochen) bzw. 0,69 (3 Wochen). Das mediane OS war noch in keiner Studiengruppe erreicht. Wie Hauschild betonte, zeigte sich der Nutzen des Anti-PD1Antikörpers in allen untersuchten Subgruppen. Sowohl unter Pembrolizumab als auch unter Ipilimumab traten immunvermittelte Nebenwirkungen auf, von denen die meisten nach Einleiten einer adäquaten medizinischen Therapie abklangen. «Die Häufigkeit von Colitis und Diarrhö liegt bei dieser Immuntherapie (Pembrolizumab) unter 10\% und ist damit deutlich seltener als unter Ipilimumab", betonte Hauschild.

\section{Laufende Studien bei vielen Tumorentitäten}

Derzeit wird die Wirksamkeit von Pembrolizumab als Monotherapie oder in Kombination mit anderen Therapien in über 100 klinischen Studien - darunter 9 Phase-III-Studien - bei mehr als 16000 Patienten mit über 30 Tumorentitäten untersucht.

Wie Hauschild berichtete, gibt es speziell im Bereich der Dermatologie bereits erste vielversprechende Daten bei Patienten mit malignem Melanom und Hirnmetastasen sowie bei Patienten mit uvealen und desmoplastischen Melanomen. Spannend findet Hauschild auch den Ansatz der PN029-Studie, in der die Wirksamkeit von Pembrolizumab in Kombination mit Interferon- $\alpha$ bei Melanompatienten getestet wird. Es gebe Hinweise darauf, dass Interferon- $\alpha$ Zielmoleküle von Pembrolizumab hochreguliert, was möglicherweise das Therapieansprechen auf den Antikörper verbessere. Interessant sei auch die Kombination von Pembrolizumab mit T-VEC, einer Herpes-simplex-Virus-Typ-1-basierten onkolytischen Immuntherapie bei Patienten mit fortgeschrittenem malignem Melanom. Nicht zuletzt gebe es inzwischen auch Ansätze zum adjuvanten Einsatz von Pembrolizumab.

\section{Auf der Suche nach der optimalen Sequenz}

Intensiv diskutiert wird laut Hauschild derzeit die Frage, in welcher Sequenz Immuntherapeutika wie Pembrolizumab und zielgerichtete Therapeutika wie etwa BRAF-Inhibitoren bei Patienten mit bestehender BRAF-V600-Mutation eingesetzt werden. Da die Wirkung von PD1-Antikörpern wie Pembrolizumab oder Nivolumab deutlich schneller einsetzt als die Wirkung des CTLA4-Antikörpers Ipilimumab, stellt sich zunehmend die Frage, ob Patienten mit BRAF-V600-Mutation und hoher Tumorlast automatisch zuerst einen BRAF-Inhibitor erhalten sollen, dessen Wirkung erfahrungsgemäß besonders schnell einsetzt. All diese Fragen müssen laut Hauschild durch weitere Untersuchungen geklärt werden. Zudem müssten mit den neuen Medikamenten weitere Erfahrungen im klinischen Alltag gesammelt werden.

\section{Dr. Claudia Schöllmann, Grafsbrunn}

\section{Quelle}

MSD-Launchpressekonferenz Pembrolizumab (KEYTRUDA ${ }^{\circledR}$ ), München, 24. Juni 2015
Literatur
1 Robert C et al.: N Engl J Med 2015;372:2521-2532.

Weitere Informationen bei

POMME-med GmbH

Dr. Claudia Schöllmann

schoellmann@pomme-med.de

\section{PharmaTicker+++ PharmaTicker+++ PharmaTicker+++ PharmaTicker+++}

Stallergenes. Die überfällige Gleichbewertung von SCIT und SLIT ist laut dem Vizepräsident der Deutschen Gesellschaft für Allergologie und klinische Immunologie, Prof. Claus Bachert, die wichtigste Neuerung der aktuellen S2k-Leitlinie zur Spezifischen Immuntherapie. Auf Grundlage der neuen Leitlinie gab Bachert im Rahmen einer Experten-Diskussionsrunde Therapieempfehlungen für die Praxis, die vom Goldstandard bis zur nicht mehr zu empfehlenden Alternative reichten. Zwei Präparate, die den neuen Goldstandard darstellen, sind Oralair ${ }^{\circledR}$ und Staloral ${ }^{\circledR}$.

Stallergenes $\mathrm{GmbH}$ www.stallergenes.de
Medice. Bei Sonnenbrand, Sonnenallergien und entzündeten Insektenstichen sind hydrocortisonhaltige Topika nicht mehr wegzudenken. Hierbei kommt es nicht nur auf den Wirkstoff, sondern auch auf das Vehikel an. Daher gibt es das Soventol ${ }^{\circledR}$ Cremogel jetzt auch höherdosiert mit 0,5\% Hydrocortisonacetat. Die innovative Cremogel-Technologie erweist sich in Studien als galenischer Wirkverstärker, zeigt in Kombination mit Hydrocortisonacetat eine schnellere Wirkstofffreisetzung und führt im Ergebnis zu einer besseren Wirksamkeit, sogar im Vergleich mit höher dosierten Präparaten.

Medice Arzneimittel Pütter GmbH \& Co. KG Katrin Nowitzky / k.nowitzky@ medice.de
Lilly. Der an IL-17A bindende monoklonale Antikörper Ixekizumab wird derzeit zur Behandlung mittelschwerer bis schwerer Plaque-Psoriasis untersucht. Detaillierte Ergebnisse aus 2 zulassungsrelevanten Phase-III-Studien zu Ixekizumab wurden bereits in «The Lancet» veröffentlicht. In den klinischen Studien UNCOVER-2 und UNCOVER-3 mit über 2500 Patienten erwies sich Ixekizumab in allen Parametern des Hautzustands gegenüber Etanercept und Placebo als statistisch überlegen. Darüber hinaus zeigten die Patienten eine signifikante Verbesserung der Lebensqualität.

Lilly Deutschland $\mathrm{GmbH}$

Pressestelle / pressestelle @ lilly.com 


\section{PharmaNews}

Karger Kompass Dermatologie 3 | 2 | 15

Plaque-Psoriasis

\section{Begleiterkrankungen und Persistenz als wichtige Therapieparameter}

Als Systemerkrankung ist die Psoriasis mit vielen Begleiterkrankungen assoziiert. Die hieraus erwachsenden Anforderungen an den Dermatologen standen im Fokus eines Janssen-Symposiums anlässlich der 48. Tagung der Deutschen Dermatologischen Gesellschaft. Vorgestellt wurden auch Registerdaten zur Persistenz in der Plaque-Psoriasis-Therapie mit Biologika, die signifikant bessere Ergebnisse unter Stelara ${ }^{\circledR}$ (Ustekinumab) im Vergleich zu subkutanen TNF- $\alpha$-Inhibitoren aufzeigten [1].

Auf dem Mittagssymposium von Janssen verwies PD Dr. Marc Alexander Radtke, Universitätsklinikum Hamburg-Eppendorf, auf den besonderen Stellenwert der Komorbidität und forderte, die neu gewonnenen Erkenntnisse aus der Forschung in die Praxis zu überführen. Der Dermatologe nehme die Rolle eines Therapiemanagers und "Gatekeepers" ein, der die Psoriasis als eine Systemerkrankung begreifen, Begleiterkrankungen wie Psoriasis-Arthritis und kardiovaskuläre Erkrankungen berücksichtigen und in interdisziplinärer Zusammenarbeit mit Rheumatologe und Hausarzt die Versorgung der Patienten sicherstellen solle. Der Arzt müsse bei der Wahl der Psoriasis-Therapie im Blick behalten, welchen Einfluss diese auf das kardiovaskuläre Risiko bzw. die kardiovaskuläre Ereignisrate des Patienten haben kann, betonte Radtke. Bei der Behandlung der Plaque-Psoriasis mit dem Interleukin(IL)-12/IL-23-Antagonisten Ustekinumab sieht Radtke bisher keine Hinweise auf ein erhöhtes Risiko hinsichtlich schwerer kardiovaskulärer Ereignisse (MACE). In einer gepoolten Analyse von Phase-II/III-Studiendaten konnte über einen Zeitraum von 4 Jahren ein übereinstimmendes MACE-Risiko im Vergleich zur US-Bevölkerung und zu Psoriatikern in Großbritannien identifiziert werden [2].
Dr. Sascha Gerdes, Universitäts-Hautklinik Kiel, widmete sich der Adipositas, da diese ein zentraler Faktor bei Psoriasis und ihrer Pathogenese sei. In einer kontrollierten Studie aus Italien wurde gezeigt, dass Patienten durch eine Gewichtsreduktion hinsichtlich ihres Erfolges bei der Behandlung der Psoriasis profitierten [3]. Deshalb sei sie ein zweckmäßiger Bestandteil der Therapiestrategie bei Psoriasis, schlussfolgerte Gerdes. An diesem Punkt setze das von Janssen unterstützte Online-Coaching «SkinWinner» an. Das gemeinsam mit der Universitäts-Hautklinik Kiel entwickelte Programm unterstützt Patienten mit Hilfestellungen, Motivationshilfen und Erinnerungen bezüglich ihrer Ernährung dabei, ihr Körpergewicht zu reduzieren. Gerdes berichtete, dass in Kürze die Publikation von ersten Daten aus einer Pilotstudie zum «SkinWinner» mit 20 Patienten erwartet werde.

Prof. Dr. Michael Sticherling, Hautklinik Universitätsklinikum Erlangen, referierte über die Psoriasis-Therapie zwischen gestern, heute und morgen. Sticherling sah grundsätzlich 3 wichtige Säulen für die Therapie der Psoriasis: Verträglichkeit, Effektivität und eine komfortable Anwendung. Biologika wie Ustekinumab oder Adalimumab punkteten nicht nur bei der Effektivität, sondern zeigten auch eine gute Verträglichkeit und Anwenderfreundlichkeit, so der Dermatologe. Ein erreichter PASI 75 sorge dafür, dass Patienten ihre Therapie weiterführen: «Die Persistenz ist ein sehr wichtiger Aspekt, denn nur wenn Patienten eine Therapie auch langfristig anwenden, kann häufig das volle Wirkungspotenzial ausgeschöpft werden.» Register, wie das internationale PSOLAR (Psoriasis Longitudinal Assessment and Registry), bieten laut Sticherling einen Blick auf die Therapien, wie sie sich jen- seits der Studien auch im wahren Leben darstellen. PSOLAR ist eine Längsschnittstudie zur Erfassung von Verträglichkeits- und Sicherheitsdaten bei Patienten mit mittelschwerer bis schwerer Plaque-Psoriasis, die mit unterschiedlichen Arzneimitteln behandelt werden. Erste Daten wurden Ende 2014 auf dem ACR-Kongress vorgestellt. Über mindestens 4 Jahre wurde die Therapiedauer als Zeit zwischen der ersten Dosis eines Biologikums und dem Eintreten eines der folgenden Ereignisse erfasst: a) Therapiestopp, b) Biologikawechsel, c) Ausscheiden aus dem Register, d) Erreichen des letzten Datenanalyse-Zeitpunktes. Die Auswertung zeigte, dass die Persistenz bei Ustekinumab am höchsten lag. Biologikanaive Patienten wiesen demnach eine signifikant bessere Persistenz unter Ustekinumab im Vergleich zu den anderen subkutanen Biologika auf (Adalimumab vs. Ustekinumab: $\mathrm{p}<0,0001$; Etanercept vs. Ustekinumab: $p<0,0001)$. Dementsprechend war bei einer Biologika-Erstlinienanwendung die Wahrscheinlichkeit einer kontinuierlichen Therapie (gemessen als HR) deutlich erhöht, wenn mit Ustekinumab anstatt mit einem TNF- $\alpha$-Inhibitor behandelt wurde (Adalimumab vs. Ustekinumab: HR 4,99 (3,39-7,35); Etanercept vs. Ustekinumab: HR 5,59 (3,77-8,29)) [1]. Einer der Gründe für die guten Ergebnisse für Ustekinumab, so Sticherling, seien vermutlich die guten Verträglichkeitsdaten bezüglich schwerer Infekte und Malignome. Unter der Therapie mit Ustekinumab habe es bisher keine Hinweise für ein vermehrtes Auftreten gegeben [2].

\section{Literatur}

1 Menter A et al.: ACR 2014; poster 1569.

2 Reich K et al.: Br J Dermatol 2012;11:300-312.

3 Naldi L et al.: Br J Dermatol 2014;170:634-642.

Weitere Informationen bei

Janssen Cilag GmbH

Tel. +49 2137 955-955

jancil@its.jnj.com

Neue internationale S3-Leitlinie zum Handekzem: Frühe Diagnostilk und Systemtherapie im Fokus

Im Rahmen der 48. Jahrestagung der Deutschen Dermatologischen Gesellschaft (DDG) wurde die im Januar 2015 erschienene, weltweit erste internationale S3-Leitlinie zum Handekzem [1] auf einem Symposium vorgestellt: Die Implikationen für die tägliche Praxis diskutierten Professor Thomas L. Diepgen, Universitätsklinik Heidelberg, und Professor Vera Mahler, Universitätsklinik Erlangen. Klarer als in der vorherigen deutschen S1-Leitlinie [2] wird jetzt die Dauertherapie mit topischen Kortikosteroiden als empfohlene Ersttherapie im Allgemeinen auf einen Zeitraum von 6 Wochen limitiert. Als einzige Therapie unter den Systemtherapien wird das Vitamin-A-Derivat Alitretinoin $\left(\right.$ Toctino $\left.^{\circledR}\right)$ als Therapiealternative zu den topischen Kortikosteroiden mit einer 1A-Empfehlung (Evidenzniveau 1, Empfehlungsgrad A) beim schweren, chronischen Handekzem herausgestellt.

Auch die frühe Diagnostik gewinnt weiter an Gewicht: Spätestens nach 3 Monaten oder bei rezidivierendem Verlauf sollte die Rolle möglicher Kon- taktallergene mittels Epikutandiagnostik abgeklärt werden. «Ziel der neuen S3-Leitlinie ist es", so Diepgen, "dass wir schwere Hauterkrankungen - und dazu zählen wir auch das Handekzem - früh einer strukturierten Diagnostik und effektiven Systemtherapie zuführen.»

\section{Quelle}

Satelliten-Symposium «Handekzem aktuell» im Rahmen des DDG-Kongresses, 29. April 2015, Berlin: Veranstalter: GlaxoSmithKline GmbH \& Co. KG

Literatur

1 Diepgen TL et al.: J Dtsch Dermatol Ges 2015:13:e1-22.

2 Diepgen TL et al.: J Dtsch Dermatol Ges 2009;7(Suppl. 3):S1-S16.

GlaxoSmithKline GmbH \& Co. KG

Corinna Mittnacht

Corinna.C.Mittnacht@gsk.com 


\section{PharmaNews}

Karger Kompass Dermatologie 3 | 2 | 15

\section{Effektiv und patientenfreundlich}

\section{Feldtherapie bei aktinischen Keratosen mit Ingenolmebutat}

"Bislang sind wir davon ausgegangen, dass der Übergang einer aktinischen Keratose in ein Plattenepithelkarzinom über eine stufenförmig zunehmende Dysplasie in der Epidermis erfolgt", erklärte Prof. Eggert Stockfleth, Bochum, bei einem Symposium im Rahmen der 48. Jahrestagung der Deutschen Dermatologischen Gesellschaft in Berlin. Allerdings zeigten neue Daten nun, dass sich Plattenepithelkarzinome häufig direkt aus geringergradigen (Grad I) aktinischen Keratosen (AK) entwickeln [1]. Daher sollten nicht nur die höhergradigen Keratosen, sondern alle aktinischen Läsionen behandelt werden, so Stockfleth. Sehr wichtig sei hierbei eine feldgerichtete Therapie, da die AK sich durch ein Nebeneinander von klinisch sichtbaren und anfangs noch unsichtbaren Läsionen auszeichne, ergänzte Prof. Julia Welzel, Augs- burg. Eine effektive Therapieoption mit kurzer Behandlungsdauer stellt laut beiden Experten Ingenolmebutat $\left(\right.$ Picato $^{\circledR}$ ) dar, das sowohl für die Feldtherapie an Stamm und Extremitäten als auch im Bereich des Gesichts und der Kopfhaut zugelassen ist [2].

AK gelten als Carcinoma in situ und seien somit eine Frühform des hellen Hautkrebses, die unbedingt behandlungsbedürftig sei, betonte Welzel. In der Regel handelt es sich nicht um Einzelläsionen, sondern um multiple konfluierende Hautläsionen in einem größeren Hautareal (Feldkanzerisierung). Das Ziel ist es, den Übergang zu invasiven Plattenepithelkarzinomen durch eine frühzeitige Diagnose und Behandlung der AK zu verhindern. Das sei umso wichtiger, als aktuelle Studiendaten darauf hinweisen, dass sich aus proliferierenden atypischen
Basalzellen (AK Grad I) direkt Plattenepithelkarzinome entwickeln können: Dies ist bei knapp 63,8\% der AK mit Grad I der Fall [1]. Für den langfristigen Therapieerfolg sei es daher unerlässlich, alle aktinischen Läsionen zu behandeln - auch die geringgradigen und noch nicht sichtbaren, unterstrich Stockfleth. Vor diesem Hintergrund sollte unbedingt eine Feldtherapie der chronisch lichtgeschädigten Hautareale - etwa mittels medikamentöser topischer Verfahren - eingeleitet werden.

Literatur

1 Fernandez-Figueras MT et al.: J Eur Acad Dermatol Venereol 2015;29:991-997.

2 Fachinformationen Picato ${ }^{\circledR} 150 \mu \mathrm{g} / \mathrm{g}$ Gel bzw. $500 \mu \mathrm{g} / \mathrm{g}$ Gel; Stand November 2013

Weitere Informationen bei

LEO Pharma GmbH

Thomas Schick

thomas.schick@leo-pharma.com

Langzeitmanagement der topischen Psoriasis-Therapie

\section{Wie sich Adhärenz und Krankheitskontrolle im Praxisalltag verbessern lassen}

Bei Psoriasis ist ein effektives Management der topischen Langzeittherapie Voraussetzung für eine anhaltende Krankheitskontrolle. Der Schlüssel zum Erreichen dieses anspruchsvollen Therapieziels ist die Applikation von wirksamen, verträglichen und zugleich anwenderfreundlichen Topika wie Daivobet ${ }^{\circledR}$ Gel und eine proaktive Patientenführung im Sinne einer therapeutischen Allianz. Im Rahmen der 48. Jahrestagung der Deutschen Dermatologischen Gesellschaft erläuterte ausgewiesene Experten anhand neuer Studienergebnisse, wie innovative Kommunikationsinstrumente und anwenderfreundliche Galeniken die Therapietreue von Psoriasis-Patienten unterstützen können. Aufgrund einer hohen Patientenzufriedenheit ist die Gelform der Fixkombination von Calcipotriol und Betamethason in der Erhal- tungstherapie von Vorteil, wobei eine feste, 2-mal wöchentliche Anwendung höhere Remissionsraten erzielt als eine Langzeittherapie nach Bedarf. Non-Adhärenz ist eine wesentliche Herausforderung im Management der Psoriasis. Aus zahlreichen Studien geht hervor, dass bis zu 70\% der Patienten in Bezug auf die Applikation von Topika nicht adhärent sind [1]. Die Ursache für die mangelnde Therapietreue liege häufig bei der verordneten Medikation selbst, berichtete Prof. Ulrich Mrowietz, Kiel. Kosmetische Nichtakzep$\operatorname{tanz}$, Zeitaufwand und Häufigkeit der Anwendung sowie das Auftreten von Nebenwirkungen haben einen erheblichen Einfluss auf die Therapietreue $[2,3]$.

Als erste prospektive randomisierte, kontrollierte Studie überhaupt ging die PSO-TOP-Studie ge- zielt der Frage nach, welche Instrumente zur Verbesserung der Adhärenz und der Arzt-Patienten-Beziehung herangezogen werden können. "Ziel des Programms ist es, die Kommunikation zwischen Arzt und Patient zu optimieren, den Patienten ausführlich aufzuklären und ihn in die Therapieplanung einzubinden, um die Adhärenz zu verbessern", so Mrowietz.

\section{Literatur \\ 1 Augustin M et al.: Dermatology 2011;222:363-374 \\ 2 Hol K: EADV Congress 2010, poster P573. \\ 3 Zaghloul SS et al.: Arch Dermatol 2004;140:408-414.}

Weitere Informationen bei

LEO Pharma GmbH

Thomas Schick

thomas.schick@leo-pharma.com

OPDIVO ${ }^{\circledR}$ (Nivolumab) wird als erster PD-1-Inhibitor in Europa zur Behandlung

des fortgeschrittenen Melanoms zugelassen

Bristol-Myers Squibb gab am 22. Juni 2015 bekannt, dass die Europäische Kommission die Zulassung für den PD-1-Inhibitor OPDIVO ${ }^{\circledR}$ (Nivolumab) zur Behandlung von erwachsenen Patienten mit fortgeschrittenem (nichtresezierbarem oder metastasiertem) Melanom unabhängig vom BRAF-Mutationsstatus erteilt hat. Die Zulassung gilt für alle $28 \mathrm{EU}$-Mitgliedsstaaten. Sie folgte auf ein beschleunigtes Beurteilungsverfahren des europäischen Ausschusses für Humanarzneimittel (Committee for Medicinal Products for Human Use, CHMP), der am 24. April 2015 ein positives Votum für Nivolumab ausgesprochen hatte. Das beschleunigte Verfahren wurde für Nivolumab - als bislang einzigen PD-1-Inhibitor in Europa - gewährt, da der Wirkstoff zu den Arzneimitteln zählt, «die für die öffentliche Gesundheit und insbesondere unter dem Aspekt einer therapeutischen Innovation von erhebli- chem Interesse sind» [1]. Nivolumab ist der erste PD-1-Inhibitor, der durch die EU-Kommission zur Behandlung einer Krebserkrankung zugelassen wurde.

\section{Quelle}

Pressemitteilung von Bristol-Myers Squibb GmbH \& Co. KGaA, 22. Juni 2015

Literatur

1 Regulation (EC) No 726/2004 of the European Parliament and of the Council vom 31. Marz 2004.

Bristol-Myers Squibb GmbH \& Co. KGaA

Ina Fürholzer

ina.fuerholzer@bms.com 
Apremilast $\left(\mathrm{Otezl}^{\circledR}\right)$ ist ein oraler niedermolekularer Wirkstoff, der die Phosphodiesterase-4 (PDE4) intrazellulär hemmt und immunmodulatorisch wirkt. Seit dem 16. Januar 2015 ist er zur Behandlung von erwachsenen Patienten mit mittelschwerer bis schwerer chronischer PlaquePsoriasis zugelassen, die auf eine andere systemische Therapie, wie Ciclosporin oder Methotrexat oder Psoralen in Kombination mit UVALicht, nicht angesprochen haben oder bei denen eine solche Therapie kontraindiziert ist oder die diese nicht vertragen haben. Zugleich ist Apremilast allein oder in Kombination mit krankheitsmodifizierenden antirheumatischen Arzneimitteln (DMARDs) indiziert zur Behandlung der aktiven Psoriasis-Arthritis (PsA) bei erwachsenen Patienten, die auf eine vorangegangene DMARD-Therapie unzureichend angesprochen oder diese nicht vertragen haben [1].

Plaque-Psoriasis und PsA sind systemische Entzündungserkrankungen mit immenser Krankheitslast und hohem ungedeckten medizinischen Bedarf, wie Prof. Dr. Diamant Thaçi, Lübeck, und Dr. Ralph von Kiedrowski, Selters, auf dem diesjährigen DDG-Kongress betonten. «Trotz zahlreicher Therapieoptionen sind viele mittelschwer bis schwer erkrankte Patienten unterversorgt", so von Kiedrowski. Laut einer aktuellen multinationalen Patientenbefragung erhalten etwa 50\% der Psoriasis-Patienten ausschließlich eine topische Therapie, obwohl eine Systemtherapie notwendig wäre [2].

Umfassende Wirksamkeit auf Haut, Juckreiz, Nägel und Gelenke

In der Zulassungsstudie ESTEEM-1 zeigte sich, dass Patienten mit Plaque-Psoriasis unter Apremilast in der Dosierung $30 \mathrm{mg}$ 2-mal täglich (BID) zu Woche 16 signifikant häufiger ein PASI-75-Ansprechen erreichten als unter Placebo (33,1 vs. 5,3\%; $\mathrm{p}<0,0001)$ [1]. Bei konstanter Apremilast-Behandlung blieb das Ansprechen meist bis Woche 52 erhalten. Darüber hinaus führte der PDE4-Inhibitor in ESTEEM-2 zu signifikanten Verbesserungen von Kopfhaut- und Nagelbeteiligungen: 44,6\% der Patienten erreichten zu Woche 16 unter Apremilast ein NAPSI-50Ansprechen (Nail Psoriasis Severity Index; Placebo: 18,7\%; $\mathrm{p}<0,0001$ ) und 40,9\% einen ScPGA-Wert von "clear» oder "minimal» (Scalp Physician Global Assessment; Placebo: 17,2\%; $\mathrm{p}<0,0001$ ) [3]. Wie ESTEEM-1 und -2 zeigten, führte Apremilast zudem bereits nach 2 Wochen zu einer deutlichen Verbesserung des Juckreizes [4]. «Dies wird von den Patienten als enorm erleichternd empfunden und gibt ihnen zusätzliches Vertrauen in die Therapie», so Thaçi.

Darüber hinaus führte Apremilast in den Phase-III-Zulassungsstudien PALACE-1 bis - 3 zu deutlichen und anhaltenden Verbesserungen diverser Krankheitszeichen und Symptome der PsA, was zur Zulassung des PDE4-Inhibitors bei PsA führte [1]. Die kombinierte $\mathrm{Zu}$ lassung ist besonders vor dem Hintergrund bedeutsam, dass rund 30\% der Psoriasis-Patienten im Krankheitsverlauf eine PsA entwickeln [5]. Thaçi empfiehlt daher, PsoriasisPatienten frühzeitig und regelmäßig auf Gelenkbeschwerden zu untersuchen. "Bei einem positiven Befund ist es mit Apremilast möglich, eine Gelenksymptomatik im Rahmen der Systemtherapie erfolgreich mitzubehandeln", so Thaçi.
Da Apremilast oral verabreicht wird, ergibt sich zusätzlich zur breiten Wirksamkeit ein vereinfachtes Therapie-Handling. Zudem zeichnete sich Apremilast in den Zulassungsstudien durch ein konsistentes und überzeugendes Sicherheitsprofil aus. Die häufigsten Nebenwirkungen waren gastrointestinale Störungen wie Diarrhö und Übelkeit, die jedoch meist nach 4 Wochen wieder verschwanden [1]. Es gab keine Häufung schwerer Infektionen, kardiovaskulärer Ereignisse oder maligner Neuerkrankungen. Auch eine Reaktivierung latenter Tuberkulosen (TBC) wurde nicht beobachtet. Ein TBC-Pre-Screening und ein routinemäßiges Labor-Monitoring sind aufgrund der vorliegenden Erkenntnisse nicht notwendig.

\section{Quelle}

Satellitensymposien "Apremilast - die neue orale Therapie in der Plaque-Psoriasis und Psoriasis-Arthritis" und "OTEZLA ${ }^{\circledR}$ (Apremilast) bei Plaque-Psoriasis: erfolgreiche Behandlung der systemischen Entzündungserkrankung in Klinik und Praxis» im Rahmen des DDG-Kongresses, 30. April und 1. Mai 2015, Berlin; Veranstalter: Celgene $\mathrm{GmbH}$

\section{Literatur}

1 Fachinformation Otezla ${ }^{\circledR}$; Stand Januar 2015.

2 Lebwohl MG et al.: J Am Acad Dermatol 2014; 70:871-881.

3 Paul C et al.: AAD 2014; Denver, CO; poster 8412: ESTEEM-2.

4 Yosipovitch G et al.: EADV 2014; Amsterdam; poster P1682: Pruritus.

5 Mease PJ et al.: J Am Acad Dermatol 2013;69:729-735.

Weitere Informationen bei dna Communications A Division of CMGRP Deutschland GmbH Johanna Pätzold jpaetzold@dna-comms.com

\section{PharmaTicker+++ PharmaTicker+++ PharmaTicker+++ PharmaTicker+++}

Fresenius Kabi. Zur Versorgung wenig bis stark exsudierender Wunden bringt Fresenius Kabi Deutschland in Kooperation mit 3M Medica den nichtklebenden Schaumverband 3M Tegaderm Foam in den Markt. Neu an dem 3M Tegaderm Foam ist die 4-Lagen-Technik. Zwei neue Lagen mit einer feuchtigkeitskontrollierenden Schicht und einem Superabsorberkissen direkt auf dem dünnen, feinporigen Polyurethanschaum, der die Wunde abdeckt, sorgen für eine schnelle Abdampfung von überschüssigem Exsudat aus dem Verband.

Circle Comm GmbH

Dr. Corinna Kolac / corinna.kolac@ circlecomm.de
Amgen. Aktuell im Journal of Clinical Oncology publizierte Daten der Phase-III-Studie OPTiM zeigen eine signifikant höhere anhaltende Ansprechrate (DRR) bei den Patienten mit nichtresezierbarem metastatischem Melanom in den Stadien IIIB, IIIC oder IV, die mit der in der Erforschung befindlichen onkolytischen Immuntherapie Talimogene Laherparepvec behandelt worden waren, im Vergleich zu den Patienten, die einen GranulozytenMakrophagen-Kolonie-stimulierenden Faktor erhalten hatten. Die Ergebnisse zeigen, dass der primäre Endpunkt der DRR erreicht wurde.

Amgen $\mathrm{GmbH}$

Fulvia Kipper / fulvia.kipper@amgen.com
Mundipharma. Der Umgang mit chronisch-entzündlichen Erkrankungen stellt eine große Herausforderung dar - für den betroffenen Patienten, aber auch den behandelnden Arzt und Angehörige. Um den Dialog zwischen Arzt und Patient zu erleichtern, hat das forschende Pharmaunternehmen Patientenbroschüren zu verschiedenen chronisch-entzündlichen Erkrankungen unter anderem für das Fachgebiet Dermatologie zur Psoriasis-Arthritis und zur Psoriasis entwickelt. Ärzte, Betroffene und Angehörige können die Borschüren direkt unter www.mundipharma.de downloaden.

Selinka/Schmitz Public Relations GmbH Nicole Zeuner / nicole.zeuner@selinka-schmitz-pr.de 


\section{PharmaNews}

Karger Kompass Dermatologie 3 | 2 | 15

Tafinlar $^{\circledR}$ und Mekinist $^{\circledR}$

\section{CHMP empfiehlt EU-Zulassung der Kombinationstherapie für Patienten mit Melanom und BRAF ${ }^{V 600}$-Mutation}

Der Ausschuss für Humanarzneimittel (CHMP) der Europäischen Arzneimittelagentur (EMA) hat im Juli 2015 die EU-Zulassung für die Kombination aus Tafinlar ${ }^{\circledR}$ (Dabrafenib) und Mekinist ${ }^{\circledR}$ (Trametinib) zur Behandlung erwachsener Patienten mit nichtresezierbarem oder metastasiertem Melanom mit einer BRAFV600 Mutation empfohlen [1].

Die CHMP-Empfehlung für Dabrafenib und Trametinib unterstreicht die Bedeutung dieser Kombination aus zielgerichteten Therapien für Patienten mit der schwersten Form von Hautkrebs. Sie basiert auf den Ergebnissen der PhaseIII-Studien COMBI-d und COMBI-v. Die Studie COMBI-d zeigte, dass mit der Kombination aus Dabrafenib und Trametinib ein statistisch signifikanter Überlebensvorteil gegenüber einer Dabrafenib-Monotherapie erreicht werden kann (Median 25,1 vs. 18,7 Monate; Hazard Ratio (HR) 0,71; 95\%-Konfidenzintervall (KI) 0,55-0,92; $\mathrm{p}=0,011)$. Von den Patienten, die Dabrafenib in Kombination mit Trametinib erhalten hatten, waren nach einem Jahr noch $74 \%$ am Leben, nach 2 Jahren $51 \%$ - gegenüber 68 bzw. $42 \%$ von denjenigen, die nur Dabrafenib erhalten hatten [2]. Die COMBI-v-Studie zeigte, dass die Kombination aus Dabrafenib und Trametinib einen statistisch signifikanten Überlebensvorteil gegenüber einer Monotherapie mit Vemurafenib erzielt (Median für die Kombination nicht erreicht vs. 17,2 Monate; HR 0,69; 95\%-KI 0,53-0,89; $\mathrm{p}=0,005)$. In der COMBI-v-Studie lag die 1-Jahres-Überlebensrate mit der Kombination aus Dabrafenib und Trametinib bei $72 \%$, mit der Vemurafenib-Monotherapie bei 65\% [3]. Die Ergebnisse zur Sicherheit waren in diesen Studien vergleichbar mit denen, die bisher mit der Kom- bination beobachtet wurden; es traten keine neuen sicherheitsrelevanten Ereignisse auf [2,3]. Die häufigsten unerwünschten Ereignisse unter der Kombinationstherapie, die in beiden Studien bei mindestens $20 \%$ der Patienten auftraten, waren Fieber (Pyrexie), Fatigue, Übelkeit, Kopfschmerzen, Schüttelfrost, Durchfall (Diarrhö), Hautausschlag, Gelenkschmerzen (Arthralgie), Erbrechen, Bluthochdruck und Husten [2, 3]. Unerwünschte Ereignisse oder Toxizitäten waren im Allgemeinen mit supportiven therapeutischen Maßnahmen, wie sie in den Fachinformationen $[4,5]$ angegeben sind, gut beherrschbar.

Die Europäische Kommission wird die Empfehlung des CHMP prüfen und ihre Zulassungsentscheidung, die für alle $28 \mathrm{EU}$-Mitgliedsstaaten sowie für Island, Norwegen und Liechtenstein gültig ist, voraussichtlich innerhalb der nächsten 3 Monate bekannt geben.

Das metastasierte Melanom ist die schwerwiegendste und lebensbedrohlichste Art von Hautkrebs [6] und mit niedrigen Überlebensraten assoziiert: Nach 5 Jahren sind von den Patienten im späten Stadium der Erkrankung noch etwa $20 \%$ am Leben [7]. Jedes Jahr werden weltweit ungefähr 200000 neue Fälle von Melanom diagnostiziert [8], von denen etwa die Hälfte $B R A F-$ Mutationen aufweist [7, 9]. Die BRAF-Mutationen lassen sich durch Gentests nachweisen, und die Ergebnisse können für die Prognose und für die Wahl der richtigen Therapie entscheidend sein [7].

\section{Die COMBI-d-Studie}

COMBI-d war eine zulassungsrelevante randomisierte, doppelblinde Phase-III-Studie, in der die Kombination des BRAF-Inhibitors Dabra- fenib und des MEK-Inhibitors Trametinib bei Patienten mit nichtresezierbarem (Stadium IIIC) oder metastasiertem (Stadium IV) BRAF-600E/ K-mutiertem Melanom der Haut mit der Kombination aus Dabrafenib und Placebo verglichen wurde. In die Studie wurden in Zentren in Australien, Europa sowie Nord- und Südamerika 423 Patienten eingeschlossen. Primärer Endpunkt dieser Studie war das durch die Prüfärzte bestimmte progressionsfreie Überleben. Sekundäre Endpunkte waren das Gesamtüberleben (OS), die Gesamtansprechrate, die Dauer des Ansprechens und die Sicherheit. Es gab kein Cross-over zwischen den Therapiearmen [2].

\section{Die COMBI-v-Studie}

COMBI-v war eine offene, 2-armige Phase-IIIStudie, in der die Kombination aus Dabrafenib und Trametinib bei erwachsenen Patienten mit BRAF-V600-mutiertem, nichtresezierbarem oder metastasiertem Melanom mit einer VemurafenibMonotherapie verglichen wurde. Primärer Endpunkt dieser Studie war das OS [3].

\section{Literatur}

1 www.ema.europa.eu/ema/index.jsp?curl=pages/medicines/ human/medicines/002604/smops/Positive/human smop_000861.jspermid=WC0b01ac058001d127 (letzter Zugriff 30. Juli 2015)

2 Long GV et al.: Lancet 2015;

doi:10.1016/S0140-6736(15)60898-4.

3 Robert C et al.: N Engl J Med 2015;372:30-39.

4 Fachinformation Tafinlar ${ }^{\circledR}$ (Dabrafenib) $50 \mathrm{mg}, 75 \mathrm{mg}$ Hartkapseln; Stand Mai 2015.

5 www.accessdata.fda.gov/drugsatfda_docs/label/2014/ 204114s001lbl.pdf.

6 www.cancer.gov/research/progress/snapshots/melanoma (letzter Zugriff 8. Juni 2015).

7 www.cancer.org/acs/groups/cid/documents/webcontent/ 003120-pdf.pdf (letzter Zugriff 8. Juni 2015).

8 http://globocan.iarc.fr (letzter Zugriff 8. Juni 2015)

9 Klein O et al.: Eur J Cancer 2013; 49:1073-1079.

Weitere Informationen bei

Novartis Pharma GmbH

Ina Meyer

ina.meyer@novartis.com

Pierre Fabre Dermatologie:

Neue Homepage unterstützt Dermatologen bei der Patientenaufklärung

Bei Problemen mit Haut, Haaren und Nägeln ist die Diagnose meist rasch gestellt. Doch viele Patienten wünschen sich neben sicheren und wirksamen Präparaten auch praktische Tipps zum Umgang mit ihrem Leiden. Hier besteht in der ärztlichen Praxis enormer Beratungsbedarf. Wertvolle Tools für die Beratung und viele gute Hinweise bietet die neue Website www.pierrefabre-dermatologie.de.

Seit mehr als 50 Jahren ist die Entwicklung wirksamer und sicherer Präparate gegen die häufigsten Hautleiden das Anliegen von Pierre Fabre Dermatologie. Fundiertes Wissen aus pharmazeutischer Forschung verbindet sich dabei mit einem großen dermokosmetischen und galenischen Erfahrungsschatz. Das Unternehmen versteht sich als Partner von Patienten, Dermatologen und Apotheken und bietet auch auf der neuen Website www.pierrefabre-dermatologie.de ein umfangreiches Angebot an Serviceleistungen und Fortbildungen.
Im Fachkreis-Bereich finden Dermatologen alle relevanten Details zu Indikation und Zusammensetzung rezeptpflichtiger Präparate - beispielsweise zu Hemangiol ${ }^{\circledR}$, dem ersten und einzigen zugelassenen säuglingsgerechten Präparat zur Behandlung proliferativer infantiler Hämangiome oder Clarelux ${ }^{\circledR}$, der schnellen und effektiven Therapie gegen Dermatosen der Kopfhaut.

Das neue Tool «lhr Hautberater» ist dagegen auch für Patienten zugänglich und kann vom behandelnden Arzt in der Sprechstunde für weitere detaillierte und gut verständliche Informationen empfohlen werden.

Pierre Fabre Dermo-Kosmetik GmbH Jechtinger Straße 13, 79111 Freiburg info.dermo_de@pierre-fabre.com 